\title{
IS THERE A THIRD INTEGRAL OF MOTION?
}

\author{
P. PIS M IS
}

Instituto de Astronomia, Universidad Nacional Autónoma de Mexico, Mexico

\begin{abstract}
It is argued that in a galaxy like ours a third integral of motion, a third independent argument in the distribution function, should exist if the potential function has to satisfy a third condition imposed on it, namely symmetry with respect to a plane. Orbit computations of single stars in a symmetric potential of the kind (Martinet and Hayli, 1971) indicate that a third integral seems to exist for Population I stars while it ceases to exist for Population II objects. This situation is explained by the author as follows. We state that a third integral should exist for all populations alike if the Boltzmann equation is interpreted within the statistical context for which it is valid. When applied to a complex system like the galaxy the third integral of the Boltzmann equation, which holds for an elementary volume in phase space, will also hold for a single particle if the latter is representative of the behavior of the element of volume as in Population I (coherent motion) whereas it will not necessarily hold for a single star of Population II; in the latter population the elementary volume, containing the same number of stars, does not represent the behavior of the element of volume during the motion of this in phase space.
\end{abstract}

\section{Reference}

Martinet, L. and Hayli, A.: 1971, Astron. Astrophys. 14, 103. 